\title{
ADAPTIVE PACKAGING SOLUTION FOR A MICROLENS ARRAY PLACED OVER A MICRO-UV-LED ARRAY
}

\author{
Markus Luetzelschwab, Dominik Weiland, Marc P. Y. Desmulliez
}

Microsystems Engineering Centre, Heriot-Watt University

School of Engineering \& Physical Sciences, Earl Mountbatten Building

Edinburgh, EH14 4AS, Scotland, UK

\begin{abstract}
In this article a versatile packaging solution is presented that allows the static and active alignment of a microlens array that is to be placed over a microUV-LED array. A modified UV-LIGA process is applied for building up the structure. For the static approach, the microlens array rests on four posts with the aim of reducing the contact area between the two parts, hence reducing the probability of vertical misalignment. The fine height adjustment is done by electroplating a certain thickness to the electrodes where the posts are being placed. Since the electrodes can be individually addressed, a possible tilt, caused by uneven post heights, can be compensated. With minimal modifications, the structure can be rendered into a dynamic alignment system, featuring actuators for vertical and lateral movement. Even though the microlens array is part of the actuator itself, it is not connected to any potential or energy sources. A magnetic actuator is proposed and partly tested that is capable of simultaneously perform a lateral movement while the vertical actuation is in progress. As a restoring means, a gel material is used as a precursor for a photo-patternable PDMS structure.
\end{abstract}

\section{Introduction}

Surface active devices (SAD) require precise positioning of their micro-optical components. This has long been being a critical issue, since it is an important factor for the interaction between optical devices and the environment $[1,2]$. Different alignment solutions have been developed to resolve some of the inherent difficulties associated with this objective $[3,4]$. The majority of these solutions make use of bulky and expensive external alignment set-up configurations $[5,6]$. They also only offer the option of static alignment, meaning that the device will be permanently fixed after having reached its specified position. Herein is presented a versatile packaging concept that allows a microlens array ( $\mu$ Lens) to be aligned either statically or dynamically over a micro-UV-LED array ( $\mu$ LED). As shown in Fig.1 both the $\mu$ Lens and the $\mu$ LED are delivered to us in chip size which complicates the packaging process significantly. 


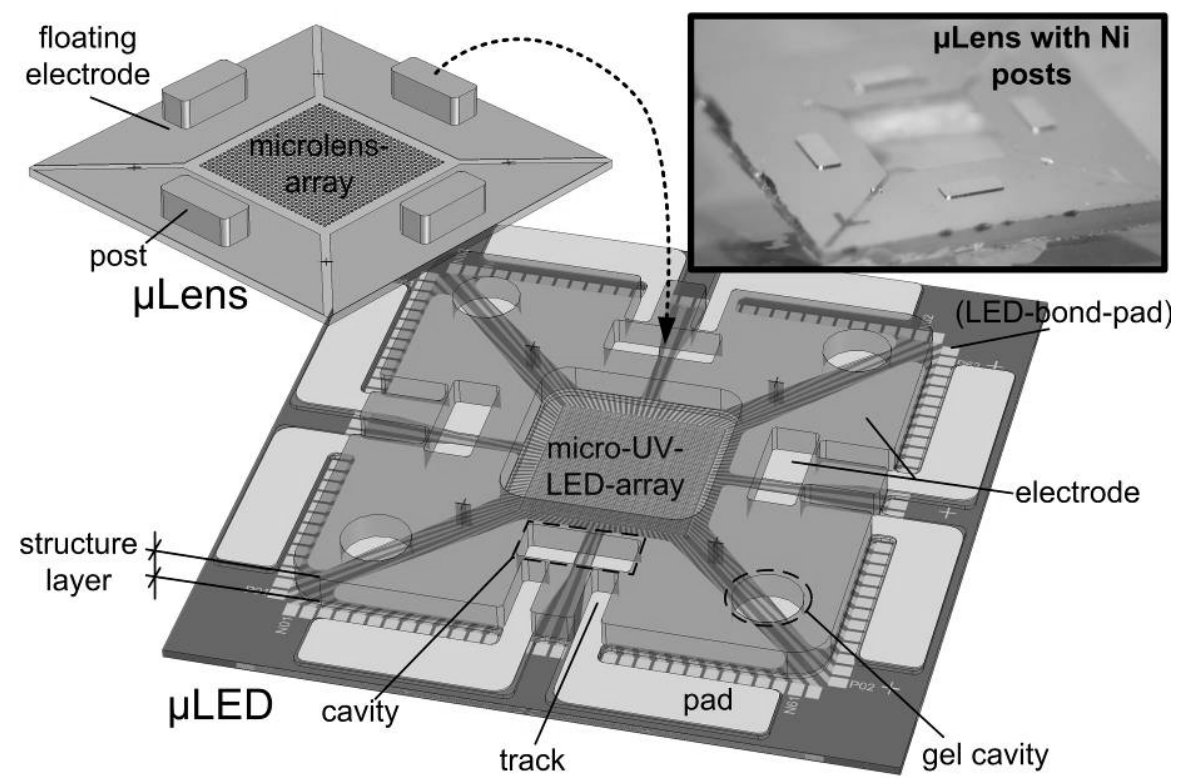

Fig. 1. The microlens array is flipped and placed on top of the micro-UV-LED array. For lateral magnetic actuation, the posts are made of a ferromagnetic material such as nickel.

The $\mu$ Lens is flipped and placed over the $\mu$ LED in order for the post to fit into the cavities. Since the posts are slightly higher than the depth of the cavities, the contact area is reduced between the two devices. This means that the probability of vertical misalignment of the whole device is reduced. With this method it is possible to "bridge" potential particulates and surface imperfections situated on the structure layer of the $\mu$ LED or the $\mu$ Lens. Each of the cavities features a pair of electrodes which can be accessed via the corresponding pads. By electroplating the electrodes to a certain thickness, vertical fine adjustment can be performed. The posts and cavities are designed to have a reduced contact area also for the lateral direction. This reduces the over determinations of the alignment process. After placing the $\mu$ Lens, the assembly can be fixed using for example UV curable adhesive. The assembly can be sealed afterwards.

With few modifications of the existing structure, the actuation of the $\mu$ Lens for dynamic alignment is possible. Electrostatic actuation in the vertical direction can be achieved as well as simultaneous lateral magnetically induced movement. Four floating electrodes are evaporated onto the $\mu$ Lens as shown in Fig.1. The purpose of the floating electrodes is to reflect the electrostatic field generated by the pair of electrodes situated underneath on the $\mu \mathrm{LED}$. No electrical potential needs to be applied to the floating electrode. No wire, which could cause mechanical disturbance, needs to be attached in consequence. Each of the four sides features an electrostatic 
actuator; therefore vertical movement as well as tilting of the $\mu$ Lens can be accomplished.

\section{Static approach}

The fabrication of the posts (Fig.1) revealed that the spin-coating of SU8 provides the specified thickness within a tolerance of around $\pm 10 \%$, giving thereby $\pm 4 \mu \mathrm{m}$ of thickness non-uniformity for an intended thickness of $40 \mu \mathrm{m}$. A method was found by fine adjusting the distance between $\mu$ LED and $\mu$ Lens caused by this tolerance by electroplating a well controlled thickness of the electrodes that are situated within the cavities of the $\mu$ LED. As shown in Fig. (top right), the overall distance between $\mu$ Lens and $\mu$ LED includes the thickness of the electrode $\left(t_{\text {elec }}\right)$ and the thickness of the post $\left(t_{\text {post }}\right)$. Since the electrodes are individually addressable, uneven post heights of $\mu$ Lens posts can be equalised as shown in Fig. 2 (left). This is particularly important, since the posts of the $\mu$ Lens might vary over the whole device. For instance, if post $\mathrm{C}$ of the $\mu$ Lens was found to be too small, then the electrode $\mathrm{C}$ needs to be electroplated in order to compensate for that mismatch.

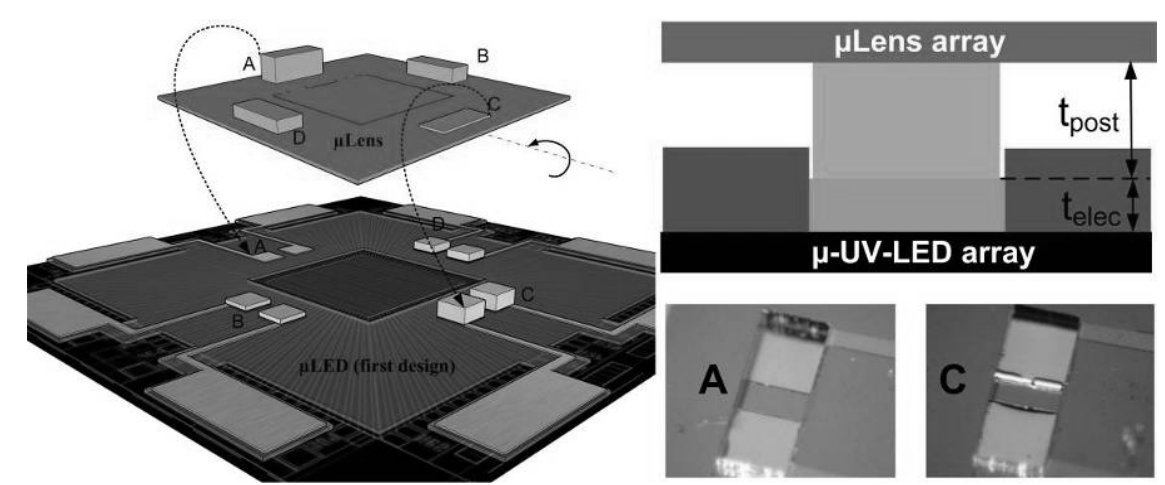

Fig. 2. Left) Different post heights on the $\mu$ Lens can be compensated. Top right) The distance between $\mu$ Lens and $\mu$ LED is comprised through the thickness of the post, $t_{\text {post }}$, and the thickness of the electrode, $t_{\text {elec. }}$.

Several electroplating tests were conducted as exampled in Fig. 3. An overall targeted thickness of $15 \mu \mathrm{m}$ is requested to level the $\mu$ Lens. The left picture in the figure is the height uniformity of the evaporated titanium electrode, taken prior to the electroplating process. The peaks are artefacts from previous process steps and are not considered in the following discussion. It is however assumed that due to the small lateral dimensions, the peaks would not contribute to a displacement with their full height, since they would probably partly deform or penetrate the SU8 post during the assembly process. The right hand side of Fig. 3 displays the situation after electroplating a nickel layer of approximately $5 \mu \mathrm{m}$. The resulting surface yields 
a thickness between 15.1 and $15.6 \mu \mathrm{m}$ and is therefore within the sub-micron range specification as far as the variation of thickness is concerned. The calculation for the required thickness was conducted by firstly determine the grow rate for a given geometry and parameters and then extrapolating the required time to achieve the intended thickness in a following electroplating process.

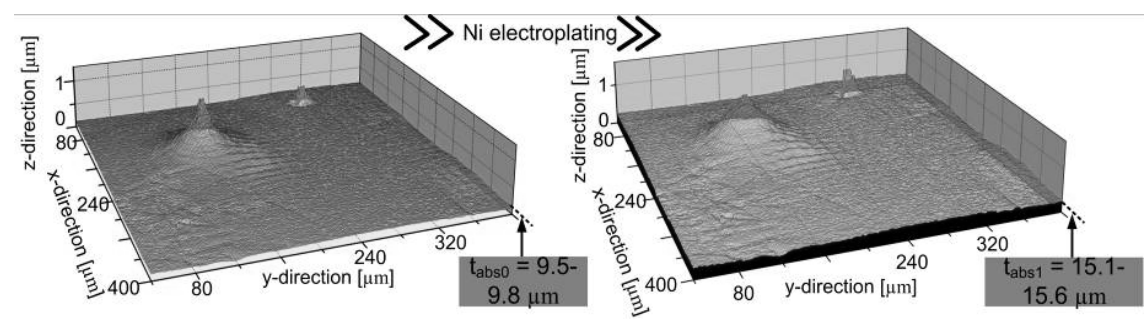

Fig. 3. Left) Height distribution of the surface of the titanium electrode with the z-axis highly exaggerated. The two peaks that are seen are due to impurities of the sample. Right) Surface height distribution of the electrode after electroplating approximately $5 \mu \mathrm{m}$ of nickel.

For the measurements shown in Fig. 3, the thickness was achieved with only one measurement for determining the grow rate. Higher accuracy could be achieved by carrying out several measurements, to approximate the target thickness very slowly at the end of the plating process.

\section{Dynamic Approach}

With minor modifications, the positioning of the $\mu$ Lens can also be dynamic and be monitored in real-time in the lateral and vertical directions. The electrodes shown in Fig. 1 then serve as a means to generate an electrostatic field [7]. The floating electrode, which is attached to the $\mu$ Lens, reflects the electrostatic field from one electrode to the other so that the assembly forms a capacitor (Fig. 4, top right). The floating electrode and hence the $\mu$ Lens does not need to be connected by any wire since no potential is required. This eases the fabrication and assembly process and frees the $\mu$ Lens from any possible mechanical disturbance caused by a wire during its movement. The FEM simulation of the force and analytical calculation curves agree with an error of less than $17 \%$ as shown in Fig. 4 on the left hand side. 


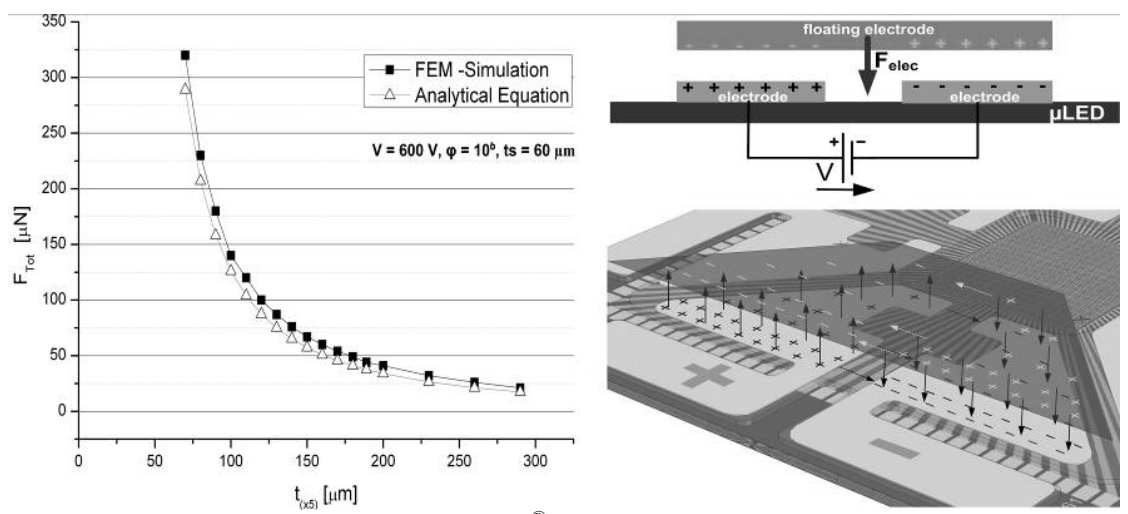

Fig. 4. Left) Analytical and FEM $\left(\mathrm{COMSOL}^{\circledR}\right)$ simulation solutions of the force generated as a function of the displacement of the electrode. Top right) Schematic of the $\mu$ Lens floating electrode forms a capacitance with the $\mu \mathrm{LED}$ electrodes. Bottom right) 3 -D representation of the figure above.

As a way to restore the $\mu$ Lens back to its initial position, gel bumps are placed within the circular cavities (Fig. 1) as shown in Fig. 5 (left) and the $\mu$ Lens is then flip-chipped on top. In the future the gel material will be replaced by photopatternable PDMS structures as PDMS offers a greater control of the shape and height $[8,9]$. The characterisation of the bumps in terms of robustness and reproducibility of their response was investigated with long term step voltage measurements using a setup similar as shown in Fig. 5 (right), without using the PIcontroller. One sample exhibited displacements of around $25 \mu \mathrm{m}$ at a voltage of $350 \mathrm{~V}$. Long term measurements revealed that the repeatability with 12 step responses, next to other different step voltages, taken over a time period of over 15 hours was good with a standard deviation of $0.43 \mu \mathrm{m}$ at a mean value of $24.75 \mu \mathrm{m}$. By reducing the size of the gel bumps, the distance between floating electrode and the electrodes is reduced as well. Due to the nonlinear relationship between electrode-to-electrode distance and the force exerted, the voltage can then be drastically reduced to achieve the same magnitude of displacement. An advantage of using gel is the possibility to have not only elastic behaviour in the vertical but also in the lateral directions. This will be of benefit for the lateral magnetic actuator as described later. A negative side effect of using gel bumps is the difficulties that are involved when trying to reproduce similar bumps in shape and volume. Each device does exhibit different displacements as a function of the voltage due to the lack of control of depositing the gel. 

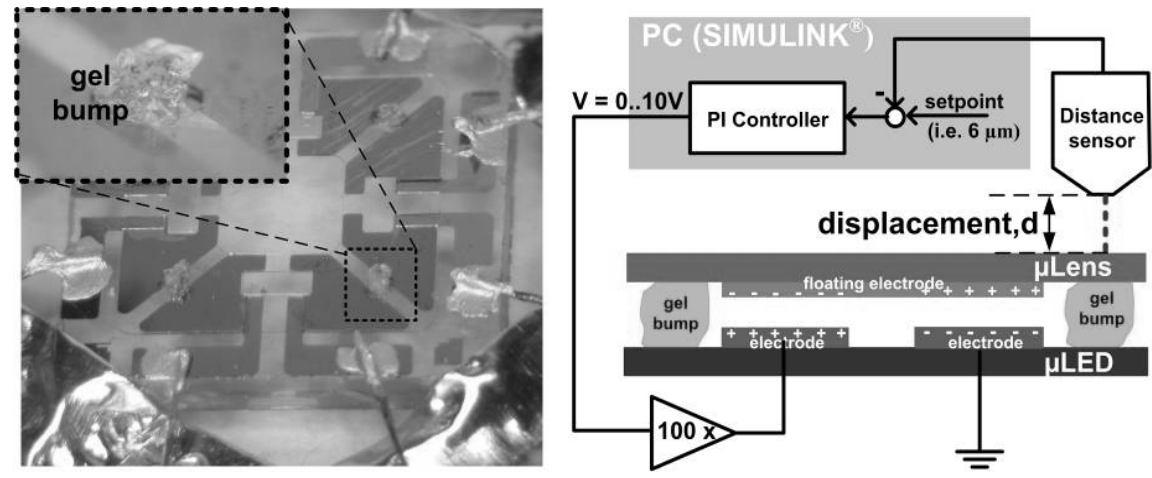

Fig.5. Left) Gel bumps, placed within circular cavities, provide the restoring force for the actuation. Right) Step response measurement setup (without PI controller) and closed control loop for accurate displacements (with PI controller).

To see whether it is possible to control the $\mu$ Lens for a certain displacement, the step response for voltages between $0 \mathrm{~V}$ and $600 \mathrm{~V}$ were recorded, as shown in Fig. 6 with a voltage on time of 2 minutes and a voltage off time of 6 minutes. The progression of the plateau of the step response, as seen in Fig.6 at time slot 1 (ts1) is not flat but with an angle $\beta$. This indicates that the restoring force does not reach an equilibrium but instead slowly continuous to increase. Furthermore, the response to the rising edge of the step is much faster compared to the falling edge. It is assumed that the stickiness of the gel and its memory properties with regard to stress and strain are the main causes of this behaviour. The mathematical description is not trivial as well as the physical effects within the system. In a first approach, the step response of the system at $400 \mathrm{~V}$ was treated as a PT2 controlled path. The slope with the angle $\beta$, was neglected. By applying a tangent to the positive slope, the dwell time, $\mathrm{t}_{\mathrm{u}}$, and compensation time, $\mathrm{t}_{\mathrm{g}}$, could be determined to be $0.25 \mathrm{~s}$ and $0.65 \mathrm{~s}$ respectively. The proportional gain (Ks) was calculated to be around 0.03 . The characteristic times and gains for the different voltages steps differ from each other so a compromise had to be taken between the obtained values. However, the dwell time, $t_{u}$, seemed to be quite consistent for the different voltage steps and even samples with a value of around $0.25 \mathrm{~s}$. From this information, the corresponding parameters were calculated with the CHR (Chien, Hrones and Reswick) method. After an initial rest run, the values needed to be changed however, since the CHR method only provides a rough approximation. The controller was chosen to be a PI controller in favor of a PID controller, since the noise of the overall system, most likely caused by the vibration of the building, would have had a disturbing effect on the differential parts of the controller. The controller was set up by using SIMULINK ${ }^{\circledR}$ and the displacement was measured by using a confocal chromatic displacement sensor (Micro-epsilon, optoNCDT 2400) as shown in Fig. 5 (right). 

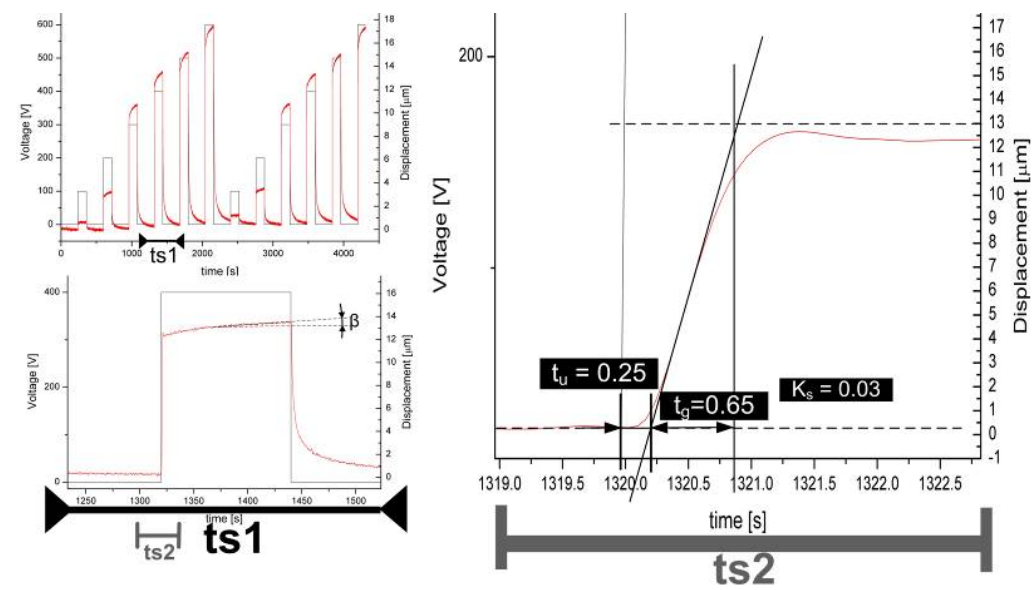

Fig.6. Top left) Step responses were recorded for voltages between 0 and 600V. Bottom left) Zoomed out picture of times slot 1 (ts1) for the $400 \mathrm{~V}$ step response. Right) Time zoom for the time slot, ts2, where different parameters were obtained.

Figure 7 depicts the approximation of the effective displacement, after changing the set point (intended displacement) discrete steps. The setpoint is achieved in approximately 25 seconds for the positive step response and around 30 to 35 seconds for the negative step response.
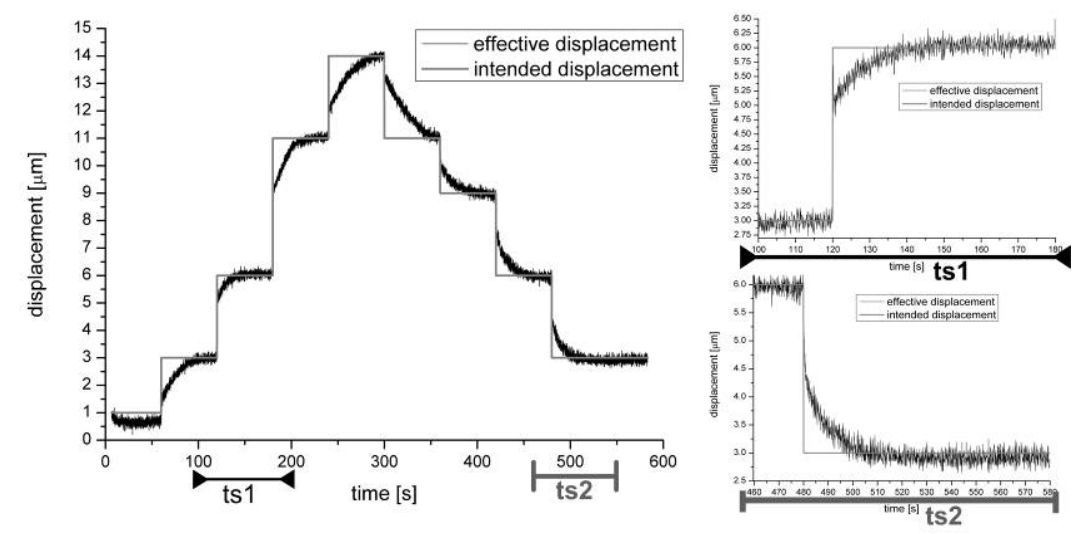

Fig. 7. Left) The setpoint of the closed control loop is changed in discrete set and the response of the system recorded. Right top and bottom) Zoomed out time slots (ts1 and ts2) from the left hand picture. 


\section{Adaptive Packaging Solution for a Microlens Array placed over a Micro-}

UV-LED Array

The noise caused by the setup and the vibration of the building makes it difficult to accurately determine the control difference that remains due to the measurement itself as well as the feedback to the system and its disturbing consequences. It is feasible to use an adaptive control loop feedback, which changes the parameters of the controller depending in which voltage range the device is operated, hence making the controlled system more responsive. The setup should be installed on top of a vibration-controlled table.

A magnetic actuator is also proposed and has been partially tested. This actuation mechanism is responsible for the lateral movement and uses the posts of the $\mu$ Lens that need to be made of a ferromagnetic material such as nickel. Each side features a magnetic actuator, thereby allowing lateral alignment of the $\mu$ Lens. The magnetic actuator does not possess a physical connection with the $\mu$ Lens which leaves the $\mu$ Lens, from a mechanical point of view, almost undisturbed. By attaching an external driving device (EDD) as shown in Fig. 8 (left), simultaneous vertical electrostatic movement and magnetic lateral movement can be performed, since the two fields can coexist within the actuator without influencing each other. The electrical insulator of the EDD needs to be robust at high voltages, but must let pass a magnetic flux that is generated by the coil with the current, $i$. The magnetic flux coming from and into the tracks (Fig.8, right bottom) attracts the Ni post with a force, $\mathbf{F}_{\mathbf{m}}$, making the distance, $\mathrm{d}_{\mathrm{m}}$, as small as possible to achieve the most flux for a given current i.
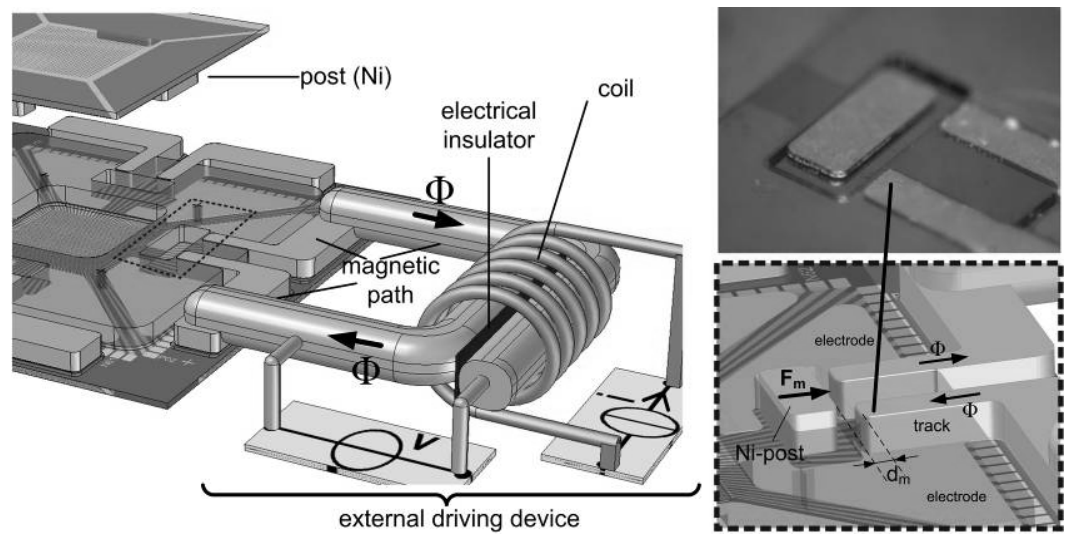

Fig. 8. Left) External driving device (EDD) attached to the ferromagnetic pads of the $\mu$ LED. The EDD allows a magnetic flux going through the magnetic path, but also provides a voltage for the electrostatic actuation. Right top) Photograph of electroplated Ni tracks leading towards the cavity where a Ni post is placed for experimental purposes. Right bottom) close up of the magnetic actuator. 
Preliminary test were conducted, and a movement of around $50 \mu \mathrm{m}$ was observed, which is well above the intended displacement range. However, a high current of $400 \mathrm{~mA}$ is necessary for this to happen and further improvement of the magnetic path is necessary. Fig. 9 displays a magnetic circuit with lumped elements where, with the exception of the magnetic resistance of air, all materials involved exhibit a nonlinear and hysteresis behaviour. In particular the latter is mathematically difficult to grasp since during operation, many different hysteresis loops are "created" while starting from different initial points. Fig. 9 (right) only depicts qualitatively the different hysteresis loops for magnetic materials that have not yet been exposed to any magnetic field. The objective is to find a MEMS process able material that exhibits high permeability but low magnetic hysteresis. With that it might be possible to represent the single magnetic resistors with lookup tables and using SIMULINK ${ }^{\circledR}$ for numerically solving the given problem within a certain degree of accuracy.

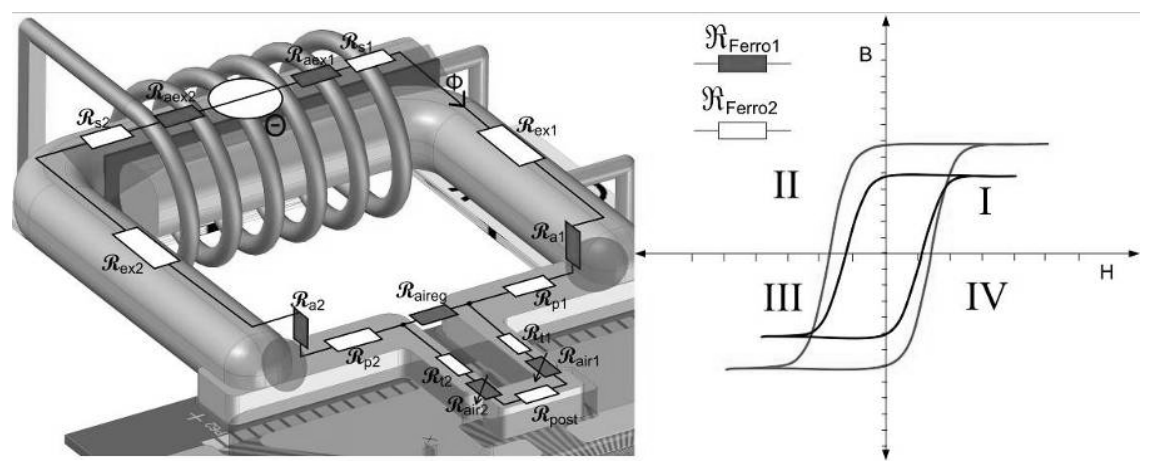

Fig. 9. Left) For illustrating purposes only, a schematic is drawn with the highly nonlinear (green and white) magnetic resistors which are supposed to have a hysteresis which is not to be neglected. Right) Schematic drawing of the magnetisation curve of the different magnetic material used.

The proposed solution with its dual actuator has the potential to have a positive impact on the production cost of such a device, in particular for the static approach. It is for instance feasible that the external driving devices are only temporarily attached to the assembly during the process of alignment and fixing in position. By doing this, the most expensive device within a production line would be 3 small intensity detectors placed closely above the $\mu$ Lens which detects the most power output, if the individual microlens is to be perfectly aligned, in vertical as well as lateral direction towards the $\mu$ LED. Three detectors would be necessary to control the $\mu$ Lens with regard to tilting and angular alignment. No expensive alignment tools are therefore necessary. It is estimated that the cost reduction of the alignment devices involved might be in the order of 10 times smaller than using a conventional alignment system. 


\section{Conclusions}

In conclusion, a device was presented that allows the accurate static alignment as well as dynamic vertical movement of a microlens array on top of a micro-UV-LED array. Both methods are capable of achieving sub micron accuracies. The use of gel bumps showed a simple and robust means for generating the necessary restoring force which will be further investigated and refined by using photo-patternable PDMS structures. Furthermore, a concept of simultaneous vertical electrostatic movement and lateral magnetic movement was proposed and partially tested.

\section{Acknowledgments}

The authors would like to express their gratitude for the financial support from the UK Engineering and Physical Science Research Council (EPSRC) through its Basic Technology Programme. The work was carried out under the project entitled "A thousand Micro-emitters per square millimeters" referenced GR/S85764.

\section{References}

[1] C. Pusarla and A. Christou, "Solder bonding alignment of microlens in hybrid receiver for free space optical interconnections," presented at Electronic Components and Technology Conference, 1996. Proceedings., 46th, 1996.

[2] S. S. Lee, L. Y. Lin, K. S. J. Pister, M. C. Wu, H. C. Lee, and P. Grodzinski, "Passively aligned hybrid integration of 8 \&times; 1 micromachined micro-Fresnel lens arrays and 8 \&times; 1 vertical-cavity surface-emitting laser arrays for free-space optical interconnect," IEEE Photonics Technology Letters, vol. 7, pp. 1031-1033, 1995.

[3] G. C. Boisset, B. Robertson, W. S. Hsiao, M. R. Taghizadeh, J. Simmons, K. Song, M. Matin, D. A. Thompson, and D. V. Plant, "On-die diffractive alignment structures for packaging of microlens arrays with 2-D optoelectronic device arrays," Photonics Technology Letters, IEEE, vol. 8, pp. 918-920, 1996.

[4] M. S. Cohen, M. J. DeFranza, F. J. Canora, M. F. Cina, R. A. Rand, and P. D. Hoh, "Improvements in index alignment method for laser-fiber array packaging," IEEE Transactions on Components, Packaging, and Manufacturing Technology Part B: Advanced Packaging, vol. 17, pp. 402-411, 1994.

[5] M. T. Gale, J. Pedersen, H. Schutz, H. Povel, A. Gandorfer, P. Steiner, and P. N. Bernasconi, "Active alignment of replicated microlens arrays on a charge-coupled device imager," Optical Engineering, vol. 36, pp. 1510-1517, 1997.

[6] S. Eitel, S. J. Fancey, H. P. Gauggel, K. H. Gulden, W. Bachtold, and M. R. Taghizadeh, "Highly uniform vertical-cavity surface-emitting lasers integrated with microlens arrays," Photonics Technology Letters, IEEE, vol. 12, pp. 459-461, 2000.

[7] S. J. Woo, J. U. Jeon, T. Higuchi, and J. Jin, "Electrostatic force analysis of electrostatic levitation system," Hokkaido, Jpn, 1995.

[8] K. M. Choi, "Photopatternable Silicon Elastomers with Enhanced Mechanical Properties for High-Fidelity Nanoresolution Soft Lithography," J. Phys. Chem. B, vol. 109, pp. 21525-21531, 2005.

[9] W. O. J.C. Loetters, P H Veltink, P Bergveld, "The mechanical properties of the rubber elastic polymer polydimethylsiloxane for sensor " Journal of Micromechanics and Microengineering, vol. 3, pp. 145-147, 1997. 